Received: 26 February 2018

Accepted: 6 November 2018

Published online: 26 November 2018

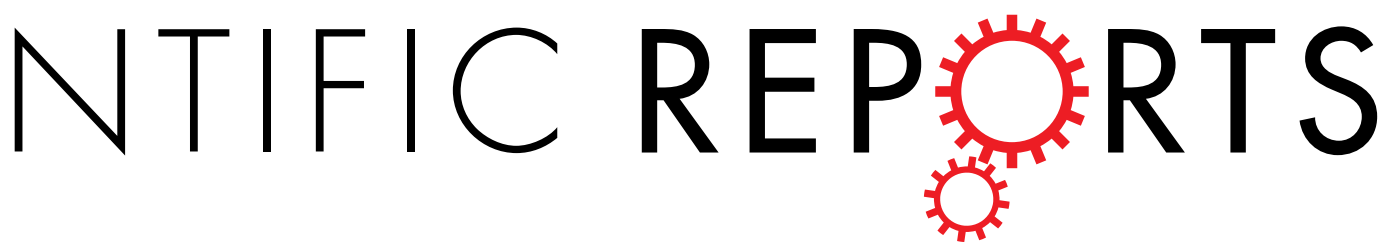

\title{
Establishing a prediction model for lateral neck lymph node metastasis in patients with papillary thyroid carcinoma
}

\section{Shan Jin, Wuyuntu Bao, Yun-Tian Yang, Tala Bai \& Yinbao Bai}

This study aimed to establish a model for predicting lateral neck lymph node metastasis in patients with papillary thyroid carcinoma. A total of 106 patients with papillary thyroid carcinoma undergoing initial treatment of neck lymph node dissection (including central and lateral neck lymph nodes) at the thyroid surgery department were enrolled from January 2009 to April 2017. Logistic regression analysis was used to screen the factors influencing lateral neck lymph node metastasis and develop a prediction model. The receiver operating characteristic curve was used to evaluate the predictive power and boundary value of the model for lateral neck lymph node metastasis. Prediction model: Logistic $(P)=-5.699+0.681 \times_{(\mathrm{TSH})}+0.342 \times_{(\text {Metastatic rate of central lymph nodes) }}+1.463 \times{ }_{(\text {Combined with Hashimoto's }}$ disease) $+1.525 \times$ (Number of tumors) . When logistic $(P)$ was $\geq 0.821$, it was predicted that lateral neck lymph node metastasis occurred in patients with papillary thyroid carcinoma. When logistic $(P)$ was $<0.821$, it was predicted that no metastasis was found in the lateral neck lymph node. The prediction accuracy was $78.3 \%$. The model helped in evaluating lateral neck lymph node metastasis in patients with papillary thyroid carcinoma. Also, it had significance in determining reasonable surgical range, reducing unnecessary lateral neck lymph node dissection, and further improving the quality of life of patients.

Thyroid cancer, a common malignant tumor of the endocrine system, has become one of the fastest-growing tumors in the world, with the incidence rate increasing year by year ${ }^{1}$. Papillary thyroid carcinoma, the most common pathological type of thyroid cancer, accounts for more than $90 \%$ of tumors, less severe malignancy. After operation and postoperative adjuvant therapy, the prognosis becomes better and long-term survival can be achieved. However, lateral neck lymph node metastasis was found in 20-90\% of patients with papillary thyroid carcinoma ${ }^{2,3}$. An important risk factor for higher recurrence rate and lower survival rate in patients with thyroid cancer is neck lymph node metastasis ${ }^{4,5}$, especially for lateral neck lymph node metastasis ${ }^{6}$. Lateral neck lymph node dissection is relatively risky and prone to accidental injury and complications because of the specificity of biological behavior of papillary thyroid carcinoma and the complexity of neck lymph node dissection. Therefore, accurate preoperative evaluation of lateral neck lymph node metastasis helps in determining the surgical range and improving tumor-free survival and quality of life of patients. The application of preoperative ultrasound, computed tomography (CT), magnetic resonance imaging (MRI), fine-needle aspiration biopsy, thyroglobulin (TG) test of puncture eluate, positron emission tomography-CT, and mutation test is still controversial in terms of the accuracy of lateral neck lymph node assessment. Combined application increases the accuracy of evaluation to some extent ${ }^{7-15}$. Therefore, predicting lateral neck lymph node metastasis in patients with papillary thyroid carcinoma needed a more objective and accurate method. In this study, logistic regression model and receiver operating characteristic (ROC) curve were used to analyze the clinicopathological features of patients with papillary thyroid carcinoma, so as to predict lateral neck lymph node metastasis. It helped in determining the surgical range, further improving the quality of life of patients and reducing recurrence. 


\begin{tabular}{|c|c|c|c|c|c|c|c|c|c|}
\hline \multirow[b]{2}{*}{ Index } & \multirow[b]{2}{*}{ Grouping } & \multicolumn{4}{|c|}{ Lateral neck lymph node metastasis } & \multirow{2}{*}{\multicolumn{2}{|c|}{ Total }} & \multirow[b]{2}{*}{ Statistics } & \multirow[b]{2}{*}{$P$ value } \\
\hline & & \multicolumn{2}{|c|}{ No } & \multicolumn{2}{|c|}{ Yes } & & & & \\
\hline Age & & 41.5 & \pm 12.4 & 41.4 & \pm 10.9 & \multicolumn{2}{|c|}{$40.6 \pm 10.5$} & 0.035 & 0.972 \\
\hline \multirow{2}{*}{ Gender } & Male & 8 & 25.8 & 17 & 22.7 & 25 & 23.6 & 0.120 & 0.729 \\
\hline & Female & 23 & 74.2 & 58 & 77.3 & 81 & 76.4 & & \\
\hline \multirow{2}{*}{ Thyroid carcinoma combined with Hashimoto's disease } & No & 24 & 77.4 & 45 & 60.0 & 69 & 65.1 & 2.929 & $0.087 * *$ \\
\hline & Yes & 7 & 22.6 & 30 & 40.0 & 37 & 34.9 & & \\
\hline \multirow{2}{*}{ Tumor location } & Left lobe & 17 & 56.7 & 21 & 41.2 & 38 & 46.9 & 1.820 & 0.249 \\
\hline & Right lobe & 13 & 43.3 & 30 & 58.8 & 43 & 53.1 & & \\
\hline \multirow{2}{*}{ Capsular invasion } & No & 28 & 90.3 & 53 & 70.7 & 81 & 76.4 & 4.702 & $0.030 *$ \\
\hline & Yes & 3 & 9.7 & 22 & 29.3 & 25 & 23.6 & & \\
\hline \multirow{2}{*}{ Multifocality } & No & 25 & 80.6 & 44 & 58.7 & 69 & 65.1 & 4.663 & $0.031 *$ \\
\hline & Yes & 6 & 19.4 & 31 & 41.3 & 37 & 34.9 & & \\
\hline \multirow{3}{*}{ Distribution of gland tumor in vivo } & Upper pole & 22 & 71.0 & 63 & 84.0 & 85 & 80.2 & & $0.219^{\#}$ \\
\hline & Middle part & 1 & 3.2 & 2 & 2.7 & 3 & 2.8 & & \\
\hline & Lower pole & 8 & 25.8 & 10 & 13.3 & 18 & 17.0 & & \\
\hline $\mathrm{T} 3$ & & \multicolumn{2}{|c|}{$1.207 \pm 0.230$} & \multicolumn{2}{|c|}{$1.190 \pm 0.226$} & \multicolumn{2}{|c|}{$1.218 \pm 0.219$} & 0.335 & 0.738 \\
\hline $\mathrm{T} 4$ & & \multicolumn{2}{|c|}{$8.502 \pm 1.548$} & \multicolumn{2}{|c|}{$8.429 \pm 1.751$} & \multicolumn{2}{|c|}{$8.631 \pm 1.660$} & 0.195 & 0.846 \\
\hline FT3 & & \multicolumn{2}{|c|}{$3.290 \pm 0.432$} & \multicolumn{2}{|c|}{$3.320 \pm 0.584$} & \multicolumn{2}{|c|}{$3.353 \pm 0.536$} & 0.259 & 0.796 \\
\hline FT4 & & \multicolumn{2}{|c|}{$1.347 \pm 0.203$} & \multicolumn{2}{|c|}{$1.503 \pm 1.805$} & \multicolumn{2}{|c|}{$1.539 \pm 1.800$} & 0.478 & 0.634 \\
\hline TSH & & \multicolumn{2}{|c|}{$2.531 \pm 1.690$} & \multicolumn{2}{|c|}{$3.253 \pm 2.146$} & \multicolumn{2}{|c|}{$2.792 \pm 1.377$} & 1.671 & $0.098^{* *}$ \\
\hline TGAb & & 59.81 & $11 \pm 102.341$ & 122. & $188 \pm 181.474$ & 94.48 & $35 \pm 143.943$ & 1.692 & $0.094 * *$ \\
\hline TPOAb & & 40.94 & $4 \pm 84.23$ & 40.9 & $3 \pm 70.45$ & 40.93 & \pm 74.81 & 0.001 & 1.000 \\
\hline Tumor diameter $(\mathrm{mm})$ & & 15.12 & $29 \pm 10.837$ & 18.0 & $10 \pm 11.261$ & 17.50 & $7 \pm 11.450$ & 1.224 & 0.224 \\
\hline Number of tumors & & 1.19 & \pm 0.40 & 1.67 & \pm 0.98 & 1.62 & \pm 0.89 & 2.600 & $0.011 *$ \\
\hline Number of central lymph node metastasis & & 2.58 & \pm 2.38 & 4.19 & \pm 3.51 & 3.72 & \pm 3.29 & 2.333 & $0.022 *$ \\
\hline Total number of central lymph node & & 7.03 & \pm 4.10 & 6.91 & \pm 3.92 & 6.98 & \pm 4.08 & 0.161 & 0.872 \\
\hline Metastatic rate of central lymph nodes & & 0.376 & $6 \pm 0.300$ & 0.63 & $3 \pm 0.360$ & 0.557 & \pm 0.363 & 3.566 & $0.001 *$ \\
\hline
\end{tabular}

Table 1. Single-factor analysis of clinicopathological results in patients with papillary thyroid carcinoma. Note: $* P<0.05$; $* * P<0.10$; ${ }^{*}$ Fisher exact probability test was adopted; Normal T3 level: $0.8-2.0 \mathrm{ng} / \mathrm{ml}$; normal T4 level: 5.1-14.1 mug/dl; normal FT3 level: 2.0-4.4 pg/mL; normal FT4 level: 0.93-1.7 ng/dL; normal TSH level: 0.2-4.2 $\mu \mathrm{IU} / \mathrm{m}$; normal TGAb level: 0-115 IU/ml; normal TPOAb level: 0-34 IU/ml;.

\section{Results}

Clinicopathological results. A total of 106 patients with papillary thyroid carcinoma were enrolled, including 25 males and 81 females with the male-to-female ratio of 1:3.24. The age range was 15-70 years, with a median age of 41.4 years. All patients underwent total thyroidectomy, as well as ipsilateral central and lateral neck lymph node dissection. The postoperative pathological results of all patients were obtained. The single-factor analysis was used to analyze clinicopathological data. Patient's gender; age; tumor location; total number of central lymph node; distribution of gland tumor in vivo; serum T3, T4, FT3, FT4, TSH, TGAb, and TPOAb levels; and tumor diameter were not found to be related to lateral neck lymph node metastasis $(P>0.05)$. However, capsular invasion, multifocality, number of tumors, and number of central lymph node metastasis were related to lateral neck lymph node metastasis, and the difference was statistically significant $(P \leq 0.05)$ (see Table 1$)$.

Factors influencing lateral neck lymph node metastasis in patients with papillary thyroid carcinoma analyzed using logistic regression. Single-factor variables with $P<0.10$, such as thyroid carcinoma combined with Hashimoto's disease, capsular invasion, multifocality, number of tumors, TSH and TGAb levels, number of central lymph node metastasis, and metastatic rate of central lymph nodes, were included in the logistic regression model. The results showed that TSH, metastatic rate of central lymph nodes, thyroid carcinoma combined with Hashimoto's disease, and number of tumors were the independent risk factors for lateral neck metastasis in patients with thyroid carcinoma $(P \leq 0.05)$. TSH increased by $1 \mu \mathrm{IU} / \mathrm{m}$, and the risk of lateral neck lymph node metastasis increased 0.975 times. The metastasis rate of central lymph nodes increased by $10 \%$, and the risk of lateral neck lymph node metastasis increased 0.407 times. The number of tumors increased by 1 , and the risk of lateral neck lymph node metastasis increased 3.595 times. The patients with thyroid carcinoma combined with Hashimoto's disease, and the risk of lateral neck lymph node metastasis increased 4.139 times (shown in Tables 2 and 3).

Establishment of prediction model. Logistic regression was used to screen the risk factors for lateral neck lymph node metastasis in patients with papillary thyroid carcinoma, and a prediction model was established. Prediction model: $\operatorname{Logistic}(\mathrm{P})=-5.699+0.681 \times_{(\mathrm{TSH})}+0.342 \times{ }_{\text {(Metastatic rate of central lymph nodes })}+1.463 \times{ }_{(\text {Combined with }}$ Hashimotoss disease) $+1.525 \times$ (Number of tumors) (TSH by laboratory examination is expressed in $\mu \mathrm{IU} / \mathrm{m}$; Metastatic rate of central lymph nodes $=$ Number of central lymph node metastasis/ Number of dissection central lymph node; 


\begin{tabular}{|c|c|c|c|c|c|c|c|c|c|}
\hline \multirow[b]{2}{*}{ Index } & \multirow[b]{2}{*}{ Grouping } & \multirow[b]{2}{*}{$\beta$} & \multirow[b]{2}{*}{ B standardization } & \multirow[b]{2}{*}{ SE } & \multirow[b]{2}{*}{ Wald $\chi^{2}$} & \multirow[b]{2}{*}{ OR } & \multicolumn{2}{|l|}{ OR 95\% CI } & \multirow[b]{2}{*}{$P$ value } \\
\hline & & & & & & & Lower limit & Upper limit & \\
\hline TSH & & 0.681 & 1.390 & 0.248 & 7.514 & 1.975 & 1.214 & 3.213 & 0.006 \\
\hline Metastasis rate of central lymph node $(10 \%)$ & & 0.342 & 1.156 & 0.101 & 11.399 & 1.407 & 1.154 & 1.716 & 0.001 \\
\hline \multirow{2}{*}{$\begin{array}{l}\text { Thyroid carcinoma combined with } \\
\text { Hashimoto's disease }\end{array}$} & No & & & & & 1 & & & \\
\hline & Yes & 1.463 & 0.701 & 0.691 & 4.486 & 4.139 & 1.115 & 16.725 & 0.034 \\
\hline Number of tumors & & 1.525 & 1.335 & 0.594 & 6.584 & 4.595 & 1.434 & 14.729 & 0.010 \\
\hline Constant term & & -5.699 & & 1.514 & 14.168 & & & & 0.001 \\
\hline
\end{tabular}

Table 2. Multivariate logistic regression analysis of lateral neck lymph node metastasis in patients with papillary thyroid carcinoma.

\begin{tabular}{|l|l|}
\hline Variable & Variable assignment specification \\
\hline Thyroid carcinoma combined with Hashimoto's disease & Yes $=1 ; \mathrm{No}=0$ \\
\hline Capsular invasion & Yes $=1 ; \mathrm{No}=0$ \\
\hline Multifocality & Yes $=1 ; \mathrm{No}=0$ \\
\hline Lateral neck lymph node metastasis & Yes $=1 ; \mathrm{No}=0$ \\
\hline & $0-10 \%=1 ; 11-20 \%=2 ; 21-30 \%=3 ; 31-40 \%=4 ;$ \\
Metastasis rate of central lymph node & $41-50 \%=5 ; 51-60 \%=6 ; 61-70 \%=7 ; 71-80 \%=8 ;$ \\
& $81-90 \%=9 ; 91-100 \%=10$ \\
\hline
\end{tabular}

Table 3. Assignment table of risk factors of lateral neck lymph node metastasis in patients with papillary thyroid carcinoma.

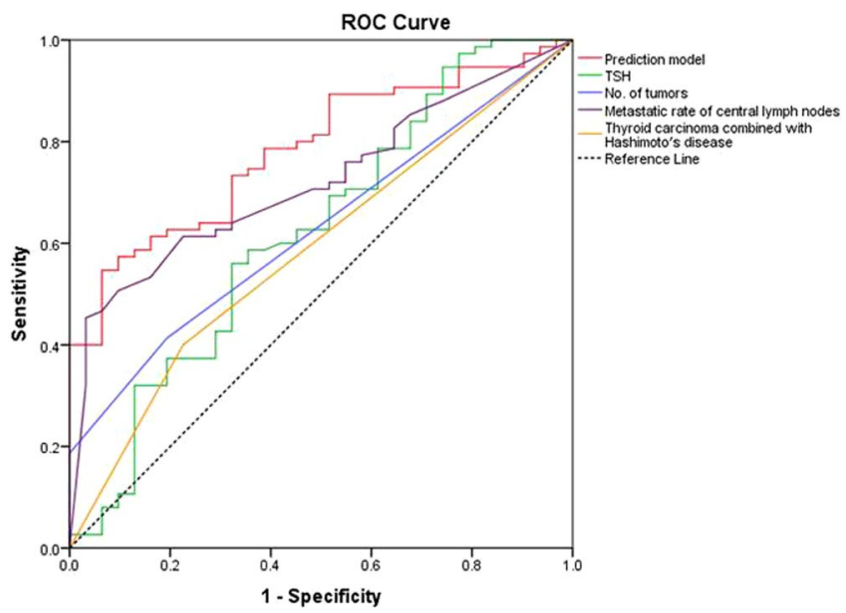

Figure 1. ROC curve for the prediction factors of lateral neck lymph node metastasis in patients with papillary thyroid carcinoma.

Combined with Hashimoto's disease: Yes $=1, \mathrm{No}=0$; Number of tumors: the number of pathologically confirmed; Number of central lymph node metastasis: the number of pathologically confirmed)(detailed model summary please see the supplementary table). ROC curve was used to evaluate the predictive power of various factors for lateral neck lymph node metastasis in patients with papillary thyroid carcinoma. TSH, thyroid carcinoma combined with Hashimoto's disease, number of tumors, metastasis rate of central lymph node, and prediction model were used as test variables. Then, ROC curve analysis was performed. The boundary value corresponding to the largest cut-off point of Youden's index was the prediction boundary value of lateral neck lymph node metastasis in patients with papillary thyroid carcinoma (shown in Fig. 1). The results showed that the AUC of prediction model was the largest ( 0.783 ; shown in Table 4$)$. The boundary value of prediction model was 0.821 , sensitivity $54.7 \%$, specificity $93.5 \%$, Youden's index $48.2 \%$, positive predictive rate $95.3 \%$, and negative predictive value $46.0 \%$. Other prediction indexes are shown in Table 5 . If logistic $(P)$ was $\geq 0.821$, it was predicted that lateral neck lymph node metastasis occurred in patients with papillary thyroid carcinoma. If logistic $(P)$ was $<0.821$, it was predicted that no metastasis was found in the lateral neck lymph node. The prediction accuracy was $78.3 \%$. 


\begin{tabular}{|l|l|l|r|l|l|l|}
\hline & & & & \multicolumn{2}{l|}{ AUC 95\% CI } \\
\cline { 7 - 8 } Index & AUC & SE & \multirow{2}{*}{ P value } & Lower limit & Upper limit \\
\hline TSH & 0.624 & 0.063 & 0.045 & 0.501 & 0.746 \\
\hline Metastasis rate of central lymph node & 0.721 & 0.050 & $<0.001$ & 0.624 & 0.818 \\
\hline Thyroid carcinoma combined with Hashimoto's disease & 0.587 & 0.059 & 0.160 & 0.471 & 0.703 \\
\hline Number of tumors & 0.628 & 0.055 & 0.039 & 0.520 & 0.736 \\
\hline Prediction model & 0.783 & 0.044 & $<0.001$ & 0.696 & 0.870 \\
\hline
\end{tabular}

Table 4. AUC of different factors in predicting lateral neck lymph node metastasis in patients with papillary thyroid carcinoma.

\begin{tabular}{|l|l|l|l|l|l|l|}
\hline Index & Cut-off & TRP & TNP & YI & PPV & NPV \\
\hline TSH & 2.495 & $56.0 \%$ & $67.7 \%$ & $23.7 \%$ & $79.2 \%$ & $37.7 \%$ \\
\hline Metastasis rate of central lymph node & $79.00 \%$ & $45.3 \%$ & $96.8 \%$ & $42.1 \%$ & $97.1 \%$ & $42.3 \%$ \\
\hline $\begin{array}{l}\text { Thyroid carcinoma combined with } \\
\text { Hashimotos disease }\end{array}$ & 0.500 & $40.0 \%$ & $77.4 \%$ & $17.4 \%$ & $81.1 \%$ & $34.8 \%$ \\
\hline Number of tumors & 1.500 & $41.3 \%$ & $80.6 \%$ & $21.9 \%$ & $83.8 \%$ & $36.2 \%$ \\
\hline Prediction model & 0.821 & $54.7 \%$ & $93.5 \%$ & $48.2 \%$ & $95.3 \%$ & $46.0 \%$ \\
\hline
\end{tabular}

Table 5. Boundary values of different factors in predicting lateral neck lymph node metastasis in patients with papillary thyroid carcinoma. Cut-off, prediction boundary value; TRP, true positive rate (sensitivity); TNP, true negative rate (specificity); YI, Youden's index; PPV, positive predictive value; NPV, negative predictive value.

\section{Discussion}

Lateral neck lymph node dissection is an important part of the treatment of papillary thyroid carcinoma. Kim et al. found that when lateral lymph node metastasis ratio $>0.3$ and largest lymph node size $>3 \mathrm{~cm}$ were prognostic factors for papillary thyroid cancer-specific mortality. Stage II patients (eighth edition) with lymph node risk (lateral lymph node metastasis ratio $>0.3$ or largest lymph node size $>3 \mathrm{~cm}$ ) had a much higher cancer-specific mortality rate (20.9\%) than those in the same stage without LN risk $(3.2 \%)^{16}$. This indicated that an important risk factor for lower survival rate in patients with thyroid cancer is neck lateral lymph node metastasis. In general, central lymph node metastasis is frequent in papillary thyroid carcinoma and is associated with lateral neck metastasis. Because of the specificity of biological behavior of papillary thyroid carcinoma, lateral lymph node skip metastasis (defined as lateral lymph node metastasis without central lymph node metastasis) is also occurred in papillary thyroid carcinoma. Chung et al. found that skip metastasis was observed in $7.7 \%$ of the papillary thyroid microcarcinoma patients ${ }^{17}$. Lateral neck lymph node dissection is prone to accidental injury and complications because of the complexity of neck dissection. The common complications include, hemorrhage, chyle leakage or lymphatic leak, nerve injury (sympathetic nerve, vagus nerve, phrenic nerve, accessory nerve, hypoglossal nerve, marginal mandibular branch of the facial nerve, brachial plexus, and cutaneous cervical plexus), incisional hydrops, incision infection, skin flap necrosis, facial swelling, and parotid gland leakage. The incidence of hemorrhage was reported as $0.29-2 \%$, chyle leakage as 6-8.3\%, sympathetic nerve injury as $5 \%$, vagus nerve injury as $0.14 \%$, phrenic nerve injury as $0.14 \%$, accessory nerve injury as $0.29-6 \%$, hypoglossal nerve injury as $0.29 \%$, injury to the marginal mandibular branch of facial nerve as $0.44 \%$, neuropathic pain of cervical plexus as $48 \%$, shoulder weakness as $27 \%$, incisional hydrops as $2 \%$, incision-related infection as $3-8 \%$, saliva leakage as $0.14 \%{ }^{18-24}$ after lateral neck lymph node dissection for treating thyroid carcinoma. This indicated that the complications of lateral neck lymph node dissection could lead to dysfunction and sometimes even affect the quality of life of patients. Therefore, it was necessary to treat each patient seriously and ensure a radical effect while protecting function and reducing the incidence of complications. In addition, the reduction of incidence of complications must be based on the reduction of unnecessary lateral neck lymph node dissection. Hence, the question was how to choose the range of lateral neck lymph node dissection in patients with papillary thyroid carcinoma in clinical practice. The American Thyroid Association (ATA), the National Comprehensive Cancer Network (NCCN), and the Chinese version of guidelines for the treatment of noncentral neck lymph node metastasis (cN1b) in patients with differentiated thyroid carcinoma recommended lateral neck lymph node dissection. A comprehensive evaluation was carried out based on the proportion of central lymph node metastasis; the location, size, and pathological typing of primary lesions of DTC; as well as the status of noncentral neck lymph node metastasis. Selective neck lymph node dissection was performed in part on patients with central neck lymph node metastasis (cN1a) $)^{25-27}$. However, the operative indications for lateral neck lymph node dissection in patients with differentiated thyroid carcinoma were not definitely given. Amin et al. suggested that clinical examinations and ultrasonography examinations are the most independent and reliable factors to detect lateral neck lymph nodes involvement ${ }^{28}$. Ultrasonography, enhanced CT, and MRI might have some significance in evaluating neck lymph nodes in the treatment of thyroid carcinoma. On the contrary, ultrasonography is related to instrument resolution, operator's experience, and degree of detail operation. Moreover, assessing parapharyngeal and level VII lymph node is not inadequate. Although CT examination overcomes the disadvantages of ultrasonography, the assessment of neck lymph node micrometastasis is poor, with the problem of contrast allergy. Enhanced MRI is affected by respiratory effects in patients and produces artifacts. Furthermore, its sensitivity is lesser compared with ultrasonography or enhanced CT. Mulla et al. analyzed the data of 5587 patients with papillary thyroid carcinoma in 19 studies. They found that the accuracy of preoperative 
ultrasonography and CT examination in assessing lateral neck lymph nodes was only $27 \%{ }^{11}$. The number of central lymph node metastasis was often used to predict whether metastasis occurred in clinical practice. Zeng et al. suggested to perform lateral neck lymph node dissection when the number of positive central lymph nodes was $\geq 2^{10}$. However, Cai et al. suggested that central lymph node metastasis in patients with papillary thyroid carcinoma was related to lateral neck metastasis. When the number of central lymph node metastasis was $\geq 3$, it could be used as a quantitative index for predicting lateral neck lymph node metastasis; also, lateral neck lymph node dissection was recommended in this case ${ }^{29}$. So et al. analyzed the data of 18,741 patients with papillary thyroid carcinoma in 23 studies. They found that the significant risk factors for lateral lymph node metastasis were not much different from known risk factors for central lymph node metastasis. Extrathyroidal extension, tumor multifocality, male sex, upper pole location, tumor size $\geq 1.0 \mathrm{~cm}$, lymphovascular invasion and tumor bilaterality were significantly associated with lateral lymph node metastasis ${ }^{30}$. Our study results showed that TSH, metastatic rate of central lymph nodes, thyroid carcinoma combined with Hashimoto's disease, and number of tumors were the independent risk factors for lateral neck metastasis in patients with thyroid carcinoma. The aforementioned studies predicted lateral neck lymph node metastasis with a single index, which lacked objectivity and comprehensiveness. Therefore, logistic regression model and ROC curve were used in this study to comprehensively analyze the clinicopathological features of patients with multiple papillary thyroid carcinomas. More risk factors were found for lateral neck lymph node metastasis in patients with papillary thyroid carcinoma. Therefore, a prediction model was developed in this study for lateral neck lymph node metastasis in patients with papillary thyroid carcinoma. The results showed that the AUC of the prediction model was the largest (0.783), positive predictive rate was $95.3 \%$, sensitivity was $54.7 \%$, specificity was $93.5 \%$, Youden's index was $48.2 \%$, and prediction accuracy was $78.3 \%$ compared with the single risk factor. This implied that the model had a better predictive ability for lateral neck lymph node metastasis in patients with papillary thyroid carcinoma compared with the single index. In clinical practice, if a high probability of lateral neck lymph node metastasis is detected in the patients by preoperative examination, the patient may undergo a surgery for the resection of lateral neck lymph nodes. However, occasionally, there is lack of evidence for the metastasis in the lateral neck lymph node by preoperative examination but pathological results confirmed the central lymph node metastasis. In this case, whether the lateral neck lymph node should be disrespected is yet to be elucidated. Thus, the present model provided a method to predict whether or not the lateral neck lymph node metastasis occurred in the non-consecutive patients. Moreover, the study aimed to reduce the unnecessary lateral neck lymph node resection, and the patients with lateral neck lymph node metastasis may early undergo reasonable treatment. In conclusion, the model provided a way to predict scientifically, quantitatively, and accurately whether lateral neck lymph node metastasis occurred in patients with papillary thyroid carcinoma. It was highly promising in determining reasonable surgical range, reducing unnecessary lateral neck lymph node dissection, and further improving the quality of life of patients.

\section{Methods}

Ethics. This study conformed to the Declaration of Helsinki regarding ethical principles for medical research in humans. It was approved by the biomedical ethics committee of Inner Mongolia Medical University (No. YKD2014063).

General data. A total of 106 initially treating patients with papillary thyroid carcinoma at the thyroid surgery department of the Affiliated Hospital of Inner Mongolia Medical University were enrolled from January 2009 to April 2017. Inclusion criteria were as follows: (1) patients with complete medical records; (2) patients who were operated by the same medical team; (3) patients who were pathologically confirmed as papillary thyroid carcinoma; (4) patients who underwent neck lymph node dissection (including central and lateral neck lymph nodes) at the same time; (5) patients without the combination with neck and other malignant tumors; (6) patients without a previous history of head and neck radiotherapy; and (7) patients without distant metastasis. Surgical procedure: Standardized surgery was performed according to the Chinese version of guidelines for "diagnosing and managing thyroid nodules and differentiated thyroid cancer" ${ }^{25}$. Patients underwent total thyroidectomy, as well as ipsilateral central and lateral neck lymph node dissection. Neck lymph node dissection: (1) central lymph node dissection (level VI): The dissection range was from the thyroid cartilage to the suprasternal fossa. The lateral border was the medial margin of carotid sheath, including anterior tracheal, paratracheal, and anterior laryngeal lymph nodes. (2) Lateral neck lymph node dissection (levels II-V): The dissection range was from digastric muscles to the clavicle. The medial border was the medial margin of the carotid sheath, and the lateral border was up to the lymph node and soft tissue of the anterior border of trapezius. Background information: Data on patient's age; gender; serum tri-iodothyronine (T3), thyroxine (T4), free triiodothyronine (FT3), free thyroxine (FT4), thyroid-stimulating hormone (TSH), antithyroglobulin antibody (TG-Ab), and thyroid peroxidase antibody (TPO-Ab) levels; size and location of primary lesions; tumor diameter; multifocality; and capsular invasion were collected. The pathological type of primary lesions, whether combined with Hashimoto's disease, and the status of neck lymph node metastasis were determined based on pathological results.

Statistical analysis. Microsoft Excel was used to organize data. SPSS 21.0 (SPSS, IL, USA) software was used for data analysis. Enumeration data were presented as the number of cases and composition ratio. Measurement data were presented as mean \pm standard deviation. The $\chi^{2}$ test, $t$ test, and Fisher's exact test were used for single-factor analysis. Nonconditional logistic regression was used for the multivariate analysis of lateral neck lymph node metastasis in patients with papillary thyroid carcinoma for the variables with $P<0.10$. The stepwise regression method was used for selecting independent variables. After ROC curve was established, the area under the curve (AUC) was calculated. The inspection level $\alpha=0.05$, and the $P$ value $\leq 0.05$ was considered statistically significant. A model for predicting lateral neck lymph node metastasis in patients with papillary thyroid carcinoma was developed based on the results of statistical analysis. 


\section{References}

1. Jin, S., Yang, Y. T. \& Bao, W. Signaling Pathways in Thyroid Cancer. Vitam Horm. 106, 501-515, https://doi.org/10.1016/ bs.vh.2017.05.002, PMID:29407446 (2018).

2. Mutalib, N. S. et al. MicroRNAs and lymph node metastasis in papillary thyroid cancers. Asian Pac J Cancer Prev. 17, 25-35 PMID:26838219 (2016).

3. Sun, W. et al. Risk Factors for central lymph node metastasis in CN0 papillary thyroid carcinoma: a systematic review and metaanalysis. PLoS. One. 10, e0139021, http://journals.plos.org/plosone/article?id=10.1371/journal.pone.0139021, (2015).

4. Grogan, R. H. et al. A study of recurrence and death from papillary thyroid cancer with 27 years of median follow-up. Surgery. 154, 1436-1447, https://doi.org/10.1016/j.surg.2013.07.008, PMID:24075674 (2013).

5. Yang, J. et al. Risk factors for level V lymph node metastases in solitary papillary thyroid carcinoma with clinically lateral lymph node metastases. Cancer Med. 5, 2161-2168, https://doi.org/10.1002/cam4.792, PMID:27368106 (2016).

6. Chéreau, N. et al. Recurrence of papillary thyroid carcinoma with lateral cervical node metastases: Predictive factors and operative management. Surgery. 159, 755-762, https://doi.org/10.1016/j.surg.2015.08.033, PMID:26435440 (2016).

7. Shin, H. J. et al. Can increased tumoral vascularity be a quantitative predicting factor of lymph node metastasis in papillary thyroid microcarcinoma? Endocrine. 47, 273-282, https://doi.org/10.1007/s12020-013-0131-8 PMID:24366642 (2014).

8. Shim, M. J. et al. Preoperative detection and predictors of level V lymph node metastasis in patients with papillary thyroid carcinoma. Br J Surg. 100, 497-503, https://doi.org/10.1002/bjs.9024, PMID:23254479 (2013).

9. Lesnik, D. et al. Papillary thyroid carcinoma nodal surgery directed by a preoperative radiographic map utilizing CT scan and ultrasound in all primary and reoperative patients. Head Neck. 36, 191-202, https://doi.org/10.1002/hed.23277, PMID:23554058 (2014).

10. Zeng. R. C. et al. Number of central lymph node metastasis for predicting lateral lymph node metastasis in papillary thyroid microcarcinoma. Head Neck. 36, 101-106, https://doi.org/10.1016/j.ejso.2012.07.119, PMID:22863305 (2014).

11. Mulla, M. G., Knoefel, W. T., Gilbert, J., McGregor, A. \& Schulte, K. M. Lateral cervical lymph node metastases in papillary thyroid cancer: a systematic review of imaging-guided and prophylactic removal of the lateral compartment. Clin Endocrinol (Oxf). 77, 126-131 https://doi.org/10.1111/j.1365-2265.2012.04336.x, PMID:22233478 (2012).

12. Liu, Z. et al. MRI and ultrasonography detection of cervical lymph node metastases in differentiated thyroid carcinoma before reoperation. Am J Transl Res. 6, 147-154 PMID:24489994 (2014).

13. Chung, J. et al. Optimal indication of thyroglobulin measurement in fine-needle aspiration for detecting lateral metastatic lymph nodes in patients with papillary thyroid carcinoma. Head Neck. 36,795-801, https://doi.org/10.1002/hed.23371, PMID:23616395 (2014).

14. Morita, S., Mizoguchi, K., Suzuki, M. \& Iizuka, K. The accuracy of 18[F]-Fluoro-2-Deoxy-D-Glucose-Positron Emission Tomography/Computed Tomography, Ultrasonography, and Enhanced Computed Tomography alone in the preoperative Diagnosis of cervical lymph node metastasis in patients with papillary thyroid carcinoma. World J Surg. 34, 2564-2569, https://doi. org/10.1007/s00268-010-0733-8, PMID:20645089 (2010).

15. Kim, S. K. et al. Role of BRAF V600E mutation as an indicator of the extent of thyroidectomy and lymph node dissection in conventional papillary thyroid carcinoma. Surgery. 158, 1500-1511 https://doi.org/10.1016/j.surg.2015.05.016, PMID:26120069 (2015).

16. Kim, H. I. et al. Refining theeighth edition AJCC TNM classification and prognostic groups for papillary thyroid cancer with lateral nodal metastasis. Oral Oncol. 78, 80-86, https://doi.org/10.1016/j.oraloncology.2018.01.021, PMID: 29496063 (2018).

17. Chung, Y. S. et al. Lateral lymph node metastasis in papillary thyroid carcinoma: results of therapeutic lymph node dissection. Thyroid. 19, 241-246 https://doi.org/10.1089/thy.2008.0244, PMID: 19265495 (2009).

18. Rotstein, L. The role of lymphadenectomy in the management of papillary carcinoma of the thyroid. J Surg Oncol. 99, 186-188, https://doi.org/10.1002/jso.21234, PMID:19170045 (2009).

19. McMullen, C., Rocke, D. \& Freeman, J. Complications of Bilateral Neck Dissection in Thyroid Cancer From a Single High-Volume Center. JAMA Otolaryngol Head Neck Surg. 143, 376-381, https://doi.org/10.1001/jamaoto.2016.3670, PMID:28097314 (2017).

20. Ardito, G. et al. Complications of neck dissections in papillary thyroid carcinoma: a modified procedure to reduce parathyroid morbidity. In Vivo. 30, 303-308, PMID:27107089 (2016).

21. Polistena, A. et al. Surgical morbidity of cervical lymphadenectomy for thyroid cancer: a retrospective cohort study over 25 years. Int J Surg. 21, 128-134, https://doi.org/10.1016/j.ijsu.2015.07.698 PMID:26253851 (2015).

22. Glenn, J. A. et al. Institutional experience with lateral neck dissections for thyroid cancer. Surgery. 158, 972-980, https://doi. org/10.1016/j.surg.2015.03.066 PMID:26187683 (2015).

23. Welch, K. \& McHenry, C. R. Selective lateral compartment neck dissection for thyroid cancer. J. Surg Res. 184, 193-199 (2013). https://doi.org/10.1016/j.jss.2013.04.084 PMID:23816244.

24. Roh, J. L., Kim, D. H. \& Park, C. I. L. Prospective identification of chyle leakage in patients undergoing lateral neck dissection for metastatic thyroid cancer. Ann Surg Oncol. 15, 424-429, https://doi.org/10.1245/s10434-007-9692-1 PMID:17999116 (2008).

25. Chinese Medical Association Surgery Society \& Chinese Medical Association Endocrine Society. Chinese Management Guidelines for Patients with Thyroid Nodules and Differentiated Thyroid Cancer. Zhongguo. Zhongliu. Linchuang. [Chinese Journal of Clinical Oncology]. 39, 1249-1272 (2012).

26. Haugen, B. R. et al. 2015 American Thyroid Association management guidelines for adult patients with thyroid nodules and differentiated thyroid cancer: the American Thyroid Association guidelines task force on thyroid nodules and differentiated thyroid cancer. Thyroid. 26, 1-133, https://doi.org/10.1089/thy.2015.0020, PMID:26462967 (2016).

27. Miccoli, P. \& Bakkar, S. Surgical management of papillary thyroid carcinoma: an overview. Updates Surg. 69, 145-150, https://doi. org/10.1007/s13304-017-0449-5. Epub2017 Apr 12 PMID:28405952 (2017).

28. Amin, A. T., Rezk, K. M. \& Atta, H. Clinical Examination and Ultrasonography as Predictors of Lateral Neck Lymph Nodes Metastasis in Primary Well Differentiated Thyroid Cancer. Journal of Cancer Therapy. 9, 55-63, https://doi.org/10.4236/ jct.2018.91007.

29. Cai, Y. et al. Number of positive central cervical lymph nodes for predicting lateral cervical lymph node metastasis in patients with papillary thyroid carcinoma. Zhonghua zhong liu za zhi [Chinese journal of oncology] 37, 628-631, PMID:26714606 (2015).

30. So, Y. K., Kim, M. J., Kim, S. \& Son, Y. I. Lateral lymph node metastasis in papillary thyroid carcinoma: A systematic review and meta-analysis for prevalence, risk factors, and location. Int J Surg. 50, 94-103, https://doi.org/10.1016/j.ijsu.2017.12.029, PMID: 29329789 (2018).

\section{Acknowledgements}

We thank Xue-Mei Wang from the School of Public Health for statistical analysis. This work is supported by the National Natural Science Foundation of China (Grant No. 81460157) and the Inner Mongolia Autonomous Region "prairie outstanding ability person" project. 


\section{Author Contributions}

B.W., B.T. and B.Y. performed the experiments, analyzed the data, and contributed equally. J.S. and Y.YT. conceived and supervised the study and wrote the manuscript.

\section{Additional Information}

Supplementary information accompanies this paper at https://doi.org/10.1038/s41598-018-35551-9.

Competing Interests: The authors declare no competing interests.

Publisher's note: Springer Nature remains neutral with regard to jurisdictional claims in published maps and institutional affiliations.

(c) (i) Open Access This article is licensed under a Creative Commons Attribution 4.0 International License, which permits use, sharing, adaptation, distribution and reproduction in any medium or format, as long as you give appropriate credit to the original author(s) and the source, provide a link to the Creative Commons license, and indicate if changes were made. The images or other third party material in this article are included in the article's Creative Commons license, unless indicated otherwise in a credit line to the material. If material is not included in the article's Creative Commons license and your intended use is not permitted by statutory regulation or exceeds the permitted use, you will need to obtain permission directly from the copyright holder. To view a copy of this license, visit http://creativecommons.org/licenses/by/4.0/.

(C) The Author(s) 2018 